\title{
Dry Acellular Oesophageal Matrix Prepared By Supercritical Carbon Dioxide
}

Alessandro Zambon ${ }^{1,2,3 \dagger}$, Massimo Vetralla ${ }^{1,2,3 \dagger}$, Luca Urbani ${ }^{4}$, Maria F. Pantano ${ }^{5}$, Giovanna Ferrentino $^{1}$, Michela Pozzobon ${ }^{6}$, Nicola M. Pugno ${ }^{5,7,8}$, Paolo De Coppi ${ }^{4,6}$, Nicola Elvassore ${ }^{2,3}$, Sara Spilimbergo ${ }^{1,2^{*}}$

${ }^{1}$ Department of Industrial Engineering, University of Trento, via Sommarive, 9 - 38123 Povo, Trento, Italy

${ }^{2}$ Department of Industrial Engineering, University of Padova, via Marzolo 9-35131 Padova, Italy

${ }^{3}$ Venetian Institute of Molecular Medicine, Via Orus Giuseppe 2- 35129 Padova, Italy

${ }^{4}$ Stem Cells and Regenerative Medicine Section, Developmental Biology and Cancer Program, Institute of Child Health, University College London - 30 Guilford Street, WC1N 1EH London, United Kingdom

${ }^{5}$ Laboratory of Bio-inspired \& Graphene Nanomechanics, Department of Civil, Environmental and Mechanical Engineering, University of Trento, Via Mesiano 77, 38123 Trento, Italy

${ }^{6}$ Stem cells and regenerative medicine lab, Foundation Institute of Pediatric Research Città della Speranza- corso stati uniti 4, 35136 Padova, Italy

${ }^{7}$ Center for Materials and Microsystems, Fondazione Bruno Kessler, Via Sommarive 18, 38123 Povo (TN), Italy

${ }^{8}$ School of Engineering and Materials Science, Queen Mary University of London, Mile End Road, London E1 4NS, U.K.

* corresponding author sara.spilimbergo@ unipd.it FAX +39049 8275461

$\dagger$ these authors have contributed equally to this work 


\section{ABSTRACT}

Recently, the use of acellular matrices in tissue engineering has become extremely significant as tissue substitute for organ/tissue reconstruction. In this clinical scenario, banking decellularised organs ready for transplantation would be mandatory for patients with emergency needs.

In this work a new process based on supercritical carbon dioxide $\left(\mathrm{SC}-\mathrm{CO}_{2}\right)$ drying technique was investigated for obtaining a dry/preserved decellularized oesophagus. Experiments were performed coupling a detergent enzymatic treatment with two different protocols: (i) $\mathrm{SC}-\mathrm{CO}_{2}$ drying; (ii) dehydration in ethanol and a subsequent $\mathrm{SC}-\mathrm{CO}_{2}$ drying. The efficiency of the treatments was investigated by monitoring the loss of weight of the treated samples and the maintenance of the extracellular matrix (ECM) architecture, composition and mechanical properties after rehydration. A successful dry acellular matrix was reached in a shorter time using the combined ethanol and SC$\mathrm{CO}_{2}$ treatment. Histological analysis reported the maintenance of the tissue matrix architecture and the collagen content for all the treated samples, while the preservation of ultrastructural features were confirmed by scanning electron microscopy (SEM). Tensile tests did not show significant differences in terms of fracture strength before and after the supercritical process. Furthermore, the scaffolds demonstrated good biocompatible properties in terms of cell culture viability in vitro. Overall, the results highlighted the potential of this novel technology to obtain a dried acellular matrix for oesophageal regeneration, preserving the extracellular matrix structure of the native tissue. 
KEYWORDS: oesophageal replacement; dry acellular matrix; supercritical carbon dioxide; supercritical drying; extracellular matrix preservation; tissue engineering; mesenchymal stem cells.

\section{INTRODUCTION}

Surgical oesophagus replacement may occur in many congenital [1] and acquired diseases [2]. Nowadays, the most commonly applied clinical treatment for oesophagus replacement is the substitution using the stomach (gastric pull-up), the colon (colonic interposition) or the small bowel, with high risks associated with morbidity and potential post-surgical complications [3-7]. Oesophageal tissue engineering (OTE) is an emerging alternative for the development of suitable tissue replacements for oesophageal repair. In the last decay, oesophageal engineered substitutes have been explored using several synthetic and natural derived scaffolds and materials [8]. Early studies seem to indicate that using synthetic material, such as silicone-based scaffolds lead to poor result in animal models $[9,10]$, while naturally derived collagen scaffolds showed promising results $[11,12]$.

Indeed, starting from the native tissue has already shown some value for clinical translation, and organ decellularization for tissue engineering has become more and more promising for the organ/tissue replacement with initial satisfactory clinical experience with simpler tabularised organs [37,38]. Organs that are more complex have also been engineered using decellularized materials in experimental animals such as heart [39] liver [13], kidney [14], intestine [40] and other organs/tissues [15-17]. Our group has recently demonstrated that a natural oesophageal acellular matrix can be also obtained directly from pig oesophagus, maintaining the ultrastructure and composition of the native extracellular matrix (ECM) [18]. These scaffolds could represent a valid therapeutic solution for the replacement of damaged organ, overcoming rejection and immunosuppression complication. Typically, natural acellular matrices from animal tissues are 
obtained through different chemical and enzymatic treatments [15-18]. Despite the safety of the procedure, detergent residua might be presented at the end of the process causing high toxicity and therefore limiting their use at clinical level [19]. Moreover, most of those processes are conducted with freshly isolated tissue that is promptly transplanted after the decellularisation/recellularization process. However, this limits the potential use in the clinical scenario where an ad hoc preparation may not always be possible, and banking decellularised organs ready for transplantation may offer advantage for patients with emergency needs. To date limited knowledge is available about the storage and preservation of these decellularized tissues. Regarding the preservation of the decellularized trachea, there is evidence that the maintenance of the decellularized scaffold is limited to few weeks after their production, requiring storage solutions and controlled temperatures [20]. Nowadays no efficient and inexpensive protocol is available for long-term maintenance of natural decellularized scaffolds. It is known that sterilization processes can significantly affect both the structure and the residual protein content [21] thus, there is a need for new conservation methods.

The drying process has a great potential as preserving option and it has already been largely used for the food products [22]. It is widely known that the residual water, or in other terms the water activity, is mostly in charge of microbial development and quality decay, while its removal ensures long term conservation.

A recent drying technique for long-term preservation in the food processing has been proposed by Brown et al. [23]. They applied $\mathrm{SC}-\mathrm{CO}_{2}$ and $\mathrm{EtOH}+\mathrm{SC}-\mathrm{CO}_{2}$ drying treatment to carrots, demonstrating that samples displayed more favourable rehydrated textural properties than air-dried equivalents, with no substantial change of product microstructure.

In the same way, $\mathrm{SC}-\mathrm{CO}_{2}$ drying treatment could allow long-life to natural acellular scaffolds, acting like a storage treatment and avoiding their rapid degradation. 
Advancements in the production and long-term preservation technology of a natural acellular matrix could be achieved by using supercritical carbon dioxide $\left(\mathrm{SC}-\mathrm{CO}_{2}\right)$ drying process which has been already investigated for food [23] with promising applications as maintenance process. $\mathrm{SC}-\mathrm{CO}_{2}$ has already shown interesting application for biomaterials on the preparation of 3D porous scaffolds and hydrogel [24-28]. Carbon dioxide $\left(\mathrm{CO}_{2}\right)$ has a moderate critical point $\left(31^{\circ} \mathrm{C}, 7.38 \mathrm{MPa}\right)$, that makes it suitable for the studies where thermal degradation must be avoided. Additionally, $\mathrm{SC}-\mathrm{CO}_{2}$ displays bactericidal properties, making it an attractive solvent for applications where sterilization steps are required [29-31].

In this work, we specifically investigated the effect of $\mathrm{SC}-\mathrm{CO}_{2}$ drying for the development of a dry natural-based engineered tissue that can be used as alternative for oesophageal substitution. To the best of our knowledge, this is the first time that $\mathrm{SC}-\mathrm{CO}_{2}$ drying technique has been applied to a natural acellular matrix obtained via DET.

We used $\mathrm{SC}-\mathrm{CO}_{2}$ drying technique on a decellularized scaffold obtained from porcine oesophagus and evaluated the morphological, mechanical and structural changing induced by the treatment. Two different experimental procedures have been explored: a single step $\mathrm{SC}-\mathrm{CO}_{2}$ drying at $35^{\circ} \mathrm{C}$ and $10 \mathrm{MPa}$ for 60 or $120 \mathrm{~min}$, and a combined double step with ethanol (EtOH) for $80 \mathrm{~min}$ followed by $\mathrm{SC}-\mathrm{CO}_{2}$ drying $\left(\mathrm{EtOH}+\mathrm{SC}-\mathrm{CO}_{2}\right)$ at $35^{\circ} \mathrm{C}$ and $10 \mathrm{MPa}$ for 15 or $30 \mathrm{~min}$. The weight was measured for both dried and rehydrated samples, followed by analysis for the evaluation of macro and micro structure, mechanical properties and ECM components. Additionally, we proved the capability of cell adhesion and viability performing a set of preliminary repopulation experiments with human bone marrow mesenchymal stem cells (hBM-MSCs) up to 7 days of culture in customized microwell. 


\section{MATERIALS AND METHODS}

\subsection{Organ harvest}

Pigs were euthanized by administration of an overdose of intravenous Pentobarbital Sodium (Sigma). Oesophagi were harvested from white domestic piglets $(n=4)$, aged from 12 to 16 weeks and weighted from 25 to $40 \mathrm{~kg}$. Following a midline incision, the oesophagus was harvested and washed with a phosphate buffer solution (PBS, Life Technology, $\mathrm{pH} 7.4$ ) containing 5\% antibiotic antimycotic solution (PBS/AA). All surgical procedures and animal handling were carried out in accordance with UK Home Office guidelines under the Animals (Scientific Procedures) Act 1986 and the local ethics committee.

\subsection{Acellular matrix generation}

Oesophageal decellularization was achieved by using a detergent enzymatic treatment (DET) following the procedure of Totonelli et al. [18]. Briefly, the oesophageal lumen was perfused by three subsequent cycles of DET using a variable speed roller pump (Masterflex L/S, Cole Palmer) at 0,6 ml/min. During each cycle, samples were immersed in 3 subsequent solutions: deionised water (resistivity $18.2 \mathrm{M} \Omega / \mathrm{CM}$ ) at $4^{\circ} \mathrm{C}$ for $24 \mathrm{~h}, 4 \%$ sodium deoxycholate (Sigma Aldrich) at room temperature for $4 \mathrm{~h}$, and 50 Kunitz units DNase-I (Sigma Aldrich) in $1 \mathrm{M} \mathrm{NaCl}$ solution (Sigma Aldrich) at room temperature for $3 \mathrm{~h}$. Planar samples (d=8mm and $\mathrm{h}=7 \mathrm{~mm}$ ) from 110 to $150 \mathrm{mg}$ weight were obtained by dissecting the oesophagus between the upper and lower sphincters on the longitudinal axis, using a $8 \mathrm{~mm}$ diameter biopsy punch (B life). Rectangular samples were obtained for the mechanical tests (see Mechanical Test section for further detail). The decellularized samples were processed and used within 2 months from the DET.

\subsection{Supercritical drying}

The supercritical drying process $\left(\mathrm{SC}-\mathrm{CO}_{2}\right)$ took place in a high pressure sapphire visualization cell (Separex S.A.S., France) with an internal volume of $50 \mathrm{ml}$, designed to resist up to $40 \mathrm{MPa}$ and 
$100^{\circ} \mathrm{C}$. A thermostatic bath and an HPLC pump (Gilson 25SC; Gilson S.A.S, France) allowed to set the internal temperature and pressure, respectively. Liquid $\mathrm{CO}_{2}$ (Messer, carbon dioxide 4.0, purity $99.990 \%$ ) was fed into the reactor by the pump with a flow rate of about $25 \mathrm{ml} / \mathrm{min}$ until reaching $10 \mathrm{MPa}$ of pressure. $\mathrm{SC}-\mathrm{CO}_{2}$ drying treatments were performed at $35^{\circ} \mathrm{C}$ and $10 \mathrm{MPa}$ : samples were treated for 60 or 120 min when only SC- $\mathrm{CO}_{2}$ was applied, while 15 or $30 \mathrm{~min}$ in case the samples were firstly dehydrated with a graded EtOH solution. The dehydration was performed dipping the samples in graded $\mathrm{EtOH}$ series solutions $(50 \%, 75 \%, 95 \%$, and 99.8\%) for $20 \mathrm{~min}$ at each step. To ensure the water and ethanol content removal, an on-off valve and a micrometric valve were concurrently used to maintain the pressure at a constant level and obtain a semi-continuous flow. Furthermore, these valves allowed the depressurization of the system in a range of 1 min to $60 \mathrm{~min}$ at the end of the process. To avoid any microbial contamination during the opening and sampling operation, a Bunsen burner flame was placed near the reactor chamber and the treated samples were collected with sterile tweezers in sterile tubes. The vessel was cleaned and disinfected with EtOH (96\% v/v, Sigma Aldrich) after each sampling. Supplementary Fig.1 shows a picture of the experimental set up for the $\mathrm{SC}-\mathrm{CO}_{2}$ drying.

\subsection{Sample weight analysis}

Samples weights were measured before and after each treatment using a microbalance (OHAUS® Explorer, Nänikon, $\mathrm{CH})$. It was assumed that all weight loss was due to the humidity removal. The weight reduction was expressed as the ratio of $\% \mathrm{~W}_{\text {end }} / \mathrm{W}_{\text {start }}$ where $\mathrm{W}_{\text {end }}$ and $\mathrm{W}_{\text {start }}$ were respectively the weight of the sample before and after the treatment. As control, we also carried out a vacuum drying process for the untreated and treated samples. The vacuum drying system consisted of a vacuum chamber, a mechanical vacuum pump and a condensate trap (cooled in liquid nitrogen) that were used to remove and trap the humidity. Reduction of the pressure to $10^{-4} \mathrm{MPa}$ allowed the liquid to evaporate at room temperature, while a nitrogen flow into the vacuum chamber allowed the samples to be dried in the absence of humid air for the entire time of the 
treatment. Statistical significance was evaluated with a post hoc ANOVA and Bonferroni correction.

Rehydration efficiency was calculated as weight recover. Dry samples were rehydrated in milliQ water at room temperature. All the mechanical and structural tests were performed on rehydrated samples placed overnight in milliQ water. All the additional investigations on the scaffold property were evaluated using 120 min drying for the SC-CO2 treatment, while in the combined treatment $\mathrm{EtOH}+\mathrm{CO} 2$ the timing was $80 \mathrm{~min}$ and $15 \mathrm{~min}$ respectively for each step.

\subsection{SEM analysis and Porosity calculation}

Samples were fixed in $2.5 \% \mathrm{v} / \mathrm{v}$ glutaraldehyde in $0.1 \mathrm{M}$ phosphate buffer and left for $24 \mathrm{~h}$ at $4{ }^{\circ} \mathrm{C}$. Following washing with $0.1 \mathrm{M}$ phosphate buffer, samples were cut into segments of approximately $0.5 \mathrm{~cm}$ length and cryoprotected in $25 \%$ sucrose, $10 \%$ glycerol in $0.05 \mathrm{M} \mathrm{PBS}(\mathrm{pH} 7.4)$ for $2 \mathrm{~h}$, then fast frozen in nitrogen slush and fractured. Samples were then placed back into the cryoprotectant at room temperature and allowed to thaw. After washing in $0.1 \mathrm{M}$ phosphate buffer ( $\mathrm{pH} 7.4)$, the material was fixed in $1 \%$ OsO4/0.1 $\mathrm{M}$ phosphate buffer $(\mathrm{pH} 7.3)$ at $3^{\circ} \mathrm{C}$ for $1.5 \mathrm{~h}$ and washed again in $0.1 \mathrm{M}$ phosphate buffer ( $\mathrm{pH}$ 7.4). After rinsing with water, specimens were dehydrated in a graded ethanol-water series to $100 \%$ ethanol, critical point dried using $\mathrm{CO}_{2}$ and finally mounted on aluminum stubs using sticky carbon taps. The fractured material was mounted to present fractured surfaces across the parenchyma to the beam and coated with a thin layer of $\mathrm{Au} / \mathrm{Pd}$ (approximately $2 \mathrm{~nm}$ thick) using a Gatan ion beam coater. Images were recorded with a 7401 FEG scanning electron microscope (Jeol, USA) at several magnifications.

Porosity was calculated using ImageJ software as percentage of poruses area. A minimum of 5 random 400x images per area (muscularis externa and submucosa) were collected and analyzed from 3 different samples.

\subsection{Mechanical characterization}


Tensile tests were performed using a Midi 10 electromechanical testing machine by MESSPHYSIK Material Testing. Samples were cut with regular rectangular shape (about $12 \times 9 \mathrm{~mm}^{2}$ ) and fixed to the loading frame through double adhesive tape at both ends (Supplementary Fig. 4). Tests were performed at a speed of $1 \mathrm{~mm} / \mathrm{s}$, corresponding to a strain rate of $0.06 \mathrm{~s}^{-1}-0.12 \mathrm{~s}^{-1}$ for isolated muscle samples and composite samples consisting of both epithelium and muscle. Tests have been performed with either longitudinal or transversal orientation for each sample's typology. Load per unit width was recorded as a function of strain. This choice was a consequence of the significant variability in the samples thickness, which on turn affected reliability of the stress calculation. In fact, along with the same sample, thickness could vary by even more than $50 \%$. All the tests were performed at room temperature at least in duplicate. Treated sample were subjected to EtOH (80 $\min )+\mathrm{SC}-\mathrm{CO}_{2}\left(10 \mathrm{MPa}, 35^{\circ} \mathrm{C}\right)$ followed by an overnight rehydration in milliQ water.

\subsection{Histological analysis}

The samples were frozen in 2-methyl-butane (Sigma Aldrich) cooled in liquid nitrogen, and 9- $\mu \mathrm{m}-$ thick sections were prepared onto Superfrost slides (Thermo Scientific, Rockville, MD, USA) using a cryomicrotome (Cryostat Leica 1860, Leica biosystem, MI, Italy). Tissue slides were fixed with 2\% paraformaldeihyde in PBS for 10 min and stained with Haematoxylin and Eosin (H\&E) (BioOptica, MI, Italy), Masson's trichrome (MT) (BioOptica, MI, Italy) and Alcian blue (AB) (BioOptica, MI, Italy) stains. Images were obtained using a microscope (Leica DMR, Leica microsystem, MI, Italy) equipped with the Leica Application software (LAS).

\subsection{Cell culture and scaffold repopulation}

Human bone marrow mesenchymal stem cells (hBM-MSCs) were provided by the Cell factory of Policlinico di Milano. hBM-MSCs were expanded in tissue culture T75 flask with complete medium: Mem-alpha (Life Technology, 32661), 10\% Fetal Bovine Serum (FBS, Life Technologies), 1\% Penicillin-Streptomycin (Life Technologies). Before reaching the confluence, 
cells were trypsinized with Trypsin-EDTA $0.25 \%$ (Life Technologies) and seeded into either conventional well or above the decellularized scaffold.

The dry decellularized samples, not stored in dried form for long-term, were previously rehydrated overnight in milliQ water and then washed in Mem-alpha before an overnight conditioning at $37^{\circ} \mathrm{C}$ in an incubator with $300 \mu \mathrm{L}$ of Mem-alpha for each sample. Medium was collected in order to evaluate the presence of toxic residuals. Each sample was inserted inside a customized microwell (Supplementary Fig 3 and Fig 4 for more technical details) made of 2 layers of Polydimethylsiloxane (PDMS, Dow Corning) in the ratio 1:10 (base: cure agent). A 24 well was used to place the microwell. Cells were seeded at $100 \mathrm{k} / \mu$ well in $150 \mu \mathrm{L}$ of completed medium for each microwell above the muscle layers placed in the up side of the microwell. Cells were left to adhere overnight before adding $1 \mathrm{~mL}$ of completed medium for each well. Medium was changed every 2 days up to 7 days of culture. Live and dead analysis (Life technology) were performed to evaluate the viability at the surface. At the end of the culture, samples were fixed with paraformaldehyde (PFA, Sigm-Aldrich), 4\% in PBS 1X (Life Technologies) for 30 min and then frozen as reported in the Histological analysis section.

Medium collected after matrix conditioning was used to culture 30kcells/well hBM-MSCs in 48 well. Morphological changing was checked daily and live and dead analysis were also performed.

\subsection{Immunofluorescence analysis}

Human protein marker was verified by immunofluorescence analysis for SUN1 while cell proliferation for ki-67. Briefly, samples slices were fixed with 2\% PFA (Sigma-Aldrich) for 10 min, permeabilized with $0.5 \%$ Triton X-100 (Sigma-Aldrich) and blocked in 2\% horse serum (Life Technologies) for $45 \mathrm{~min}$ at room temperature. The rabbit monoclonal primary antibody against SUN1 (Sigma-Aldrich) was applied overnight at room temperature, followed by incubation with the Alexa594 fluorescence-conjugated anti-rabbit IgG secondary antibody (Life Technologies) for 1 
hour at $37^{\circ} \mathrm{C}$. Nuclei were counterstained with Hoechst (Life Technologies) while cytoplasm was marked by incubation $1 \mathrm{~h}$ at $37^{\circ} \mathrm{C}$ with Phalloidin (Life Technologies). The mouse monoclonal primary antibody against $\mathrm{Ki}-67$ (Sigma-Aldrich) was applied for $1 \mathrm{~h}$ at $37^{\circ} \mathrm{C}$, followed by incubation with the Alexa594 fluorescence-conjugated anti-mouse IgG secondary antibody (Life Technologies) for 1 hour at $37^{\circ} \mathrm{C}$. Nuclei were counterstained with Hoechst (Life Technologies). After staining, slices were mounted in HI-MO (Lab-Optica) and analysed with the inverted microscope LeicaDMI6000B equipped with a mercury short-arc reflector lamp (Leica, Germany) or Leica TCS SP5 confocal microscope.

\section{RESULTS}

\subsection{Generation of a dry acellular matrix}

Dry acellular matrices were produced using two different methods, $\mathrm{SC}-\mathrm{CO}_{2}$ alone and a combined double step of ethanol $(\mathrm{EtOH})$ followed by $\mathrm{SC}-\mathrm{CO}_{2}$ drying $\left(\mathrm{EtOH}+\mathrm{SC}-\mathrm{CO}_{2}\right)$ as shown in Table 1. For drying with pure $\mathrm{CO}_{2}\left(\mathrm{~T}_{1}\right.$ and $\left.\mathrm{T}_{2}\right)$, a significant effect of the treatment time on the drying efficiency, in terms of weight reduction, was evident causing about $63 \%$ weight reduction in 60 min and about 90\% weight reduction in 120 min, respectively (Figure 1A). When dried first with EtOH for $80 \min \left(\mathrm{T}_{3}\right.$ and $\left.\mathrm{T}_{4}\right)$, a significant reduction in weight of about $90 \%$ compared to $\mathrm{SC}-\mathrm{CO}_{2}$ alone was observed after only $15 \mathrm{~min}$ of treatment (Figure 1B). This result could be easily explained as a pre-treatment of $\mathrm{EtOH}$ for $80 \mathrm{~min}$ was able to produce alone a weight reduction of about $55 \%$. No significant weight reduction was noticed when the $\mathrm{SC}-\mathrm{CO}_{2}$ treatment was prolonged to 30 min of treatment. The EtOH pre-treating time was selected at 80 min based on the reduction of sample weight as function of the residence time (Supplementary Fig. 3). Residence times higher than 80 min did not shown significant differences in water replacement. When samples were left overnight in EtOH solution (data not shown), a further weight loss of about $33 \%$ was observed; however this treatment time was not taken into account as a significant shrinkage of the samples occurred. 
An extensive vacuum process was applied to assess the residual water/liquid content in the samples after the drying processes. Both samples treated with $\mathrm{SC}-\mathrm{CO}_{2}$ and $\mathrm{EtOH}+\mathrm{SC}-\mathrm{CO}_{2}$, showed from 0.5 to $1 \%$ mass reduction after $12 \mathrm{~h}$ vacuum treatment, while the control, composed of a decellularized but not dried sample, resulted in a $90.5 \%$ weight reduction after $12 \mathrm{~h}$ of the single vacuum step, confirming that the complete moisture drying was basically achieved with both treatments. The rehydration capacity of the dried samples was studied in order to investigate the possibility to restore the moisture content after the drying process. As showed in Figure 1C and 1D, all the treated samples reported no differences in the total amount of moisture uptake, which ranged from about $65 \pm 3.5$ to $72.2 \pm 1.1 \%$ of the starting weight after $1 \mathrm{~h}$. Representative pictures of the samples before and after the drying process and rehydration are shown in Supplementary Figure 2. The combined $\mathrm{EtOH}+\mathrm{SC}-\mathrm{CO}_{2}(80 \mathrm{~min}+15 \mathrm{~min})$ drying ensured a greater retention of the original volume and shape compared to the drying with $\mathrm{SC}-\mathrm{CO}_{2}(120 \mathrm{~min})$. Despite this observation, no differences in weight reduction and rehydration recovery between the two different drying treatments were observed.

\subsection{Evaluation of Structural and Mechanical properties}

$\mathrm{H} \& \mathrm{E}, \mathrm{MT}$ and $\mathrm{AB}$ staining (Figure $2 \mathrm{~A}, \mathrm{~B}$ ) were performed to evaluate the maintenance of ECM architecture before and after the $\mathrm{SC}-\mathrm{CO}_{2}$ and the $\mathrm{EtOH}+\mathrm{SC}-\mathrm{CO}_{2}$ treatments compared with a decellularized control sample that were not dried. H\&E staining confirmed the absence of intact nuclei after DET and highlighted the generation of micro-porosity after $\mathrm{SC}-\mathrm{CO}_{2}$ treatment (Figure $2 \mathrm{~A})$. The generation of micro-porosity after the $\mathrm{SC}-\mathrm{CO}_{2}$ and the $\mathrm{EtOH}+\mathrm{SC}-\mathrm{CO}_{2}$ treatments occurred in absence of any visible histological damage to the EMC structure. The preservation of the oesophageal architecture was demonstrated with the presence of the three layers: mucosa, submucosa and muscular layers (Figure 2B) in all the analyzed samples. Masson's Trichrome staining pointed out the maintenance of the collagen in the lamina propria, submucosa and 
intermuscular septa of the muscularis externa. $A B$ staining did not show a further decrease of glycosaminoglycan (GAG) after the $\mathrm{SC}-\mathrm{CO}_{2}$ and the $\mathrm{EtOH}+\mathrm{SC}-\mathrm{CO}_{2}$ treatments.

The porosity of the samples was investigated through SEM analysis as a function of different depressurization times at the end of the drying processes (Fig 3). The porosity increased significantly only for the $\mathrm{EtOH}+\mathrm{SC}-\mathrm{CO}_{2}$ treatment (Fig 3C) for both muscular and submucosa layers and the highest increase was shown for the submucosa. The depressurization time did not affect the porosity of the samples measured after the treatments.

The mechanical properties in terms of strength and maximum strain did not show significant differences as reported in Fig. 4. Generally, the treated samples showed a bigger strain at fracture for both longitudinal and transversal samples compared to the fresh ones. For the composite samples (muscle + epithelium, Fig 4B) a bigger ultimate load/width was also observed for both transversal and longitudinal samples. However, although similar samples extracted from the same tissue and with the same orientation were taken into account, the biological nature of the tested samples affected the repeatability of the mechanical measurements, showing in some cases up to $100 \%$ and $80 \%$ difference in maximum load/width and elongation, respectively (data not shown).

\subsection{Scaffold biocompatibility and cell repopulation}

Biocompatibility was investigated through toxicity test evaluating alive and dead cells on the surface of samples and via conditioned medium. We demonstrated that harmful substances were absent after the $\mathrm{SC}-\mathrm{CO}_{2}$ treatment. In supplementary Fig 8 can be appreciated the toxic behaviour only with the conditioned medium with the untreated sample. Live and dead test showed higher mortality for the untreated samples compared to the treated ones after 48 hours (Supplementary Fig 8E) and the presence of few cells in culture after 5 days (Supplementary Fig 8F) for the same set of samples. These data were also validated from live and dead test on the sample surface (data from untreated sample not shown). High cell density and low mortality after 7 days of culture for the 
samples treated with the EtOH+SC-CO 2 method is evident (Fig 5A and the enlargement Fig 5B-D). Analogous results are reported in Supplementary Fig 9 for the $\mathrm{SC}-\mathrm{CO}_{2}$ treatment although variability was observed for samples derived from different animals (data not shown). Histological analysis demonstrated the presence of cells only on the surface of the samples (Fig 5E-F). The human cells were detected with specific human antibody for SUN1 as reported in Supplementary Fig 8 excluding the presence of residual nuclei from the decellularization process. Moreover, an efficient cell surface proliferation at 7 days was revealed by Ki67 staining as shown in Supplementary Fig 10.

\section{DISCUSSION}

Decellularized scaffolds, based on animal or human organs, are highly attractive in order to develop natural substitution for transplantation. In this study, we obtained a dry acellular oesophagus matrix by applying $\mathrm{SC}-\mathrm{CO}_{2}$ drying process to a decellularized oesophagus prepared by DET. We demonstrated that no significant additional reductions in weight could be reached after the achievement of about $90.5 \%$ of the native weight. After $\mathrm{SC}-\mathrm{CO}_{2}$ treatment, the amount of residual water in the moisture was comparable to the amount reached after $12 \mathrm{~h}$ of extensive vacuum treatment. A dry ECM was successfully generated in a short time using EtOH+SC-CO 2 treatment (90\% weight reduction after $15 \mathrm{~min}$ ), while $\mathrm{SC}-\mathrm{CO}_{2}$ alone induced the same weight reduction but with longer times, $120 \mathrm{~min}$. Faster processes are preferred not only for an industrial need (more sample treated in less time, less amount of reagents), but also because the contact time among the sample and chemicals is shorter and, likely, alteration of structural and composition might be reduced. The addition of EtOH as co-solvent during the process favourably influenced the solubility of polar solvent in $\mathrm{SC}-\mathrm{CO}_{2}$, including water $[32,33]$.

The process conditions $\left(10 \mathrm{MPa}\right.$ and $\left.35^{\circ} \mathrm{C}\right)$ used in this study were optimized on the base of previous researches. We have shown that above $10 \mathrm{MPa} \mathrm{CO}_{2}$ solubility is a weak function of pressure [34, 35]. We also [35] demonstrated that a pressure increase from 10 to $30 \mathrm{MPa}$ did not 
influence significantly $\mathrm{CO}_{2}$ solubility in the water contained in samples [36]. Temperature was fixed at $35^{\circ} \mathrm{C}$ to exploit $\mathrm{CO}_{2}$ properties in supercritical phase. Experiments at higher temperatures were not taken into account based on $\mathrm{CO}_{2}$ solubility, which decreases as temperature increases [34]. Moreover, higher temperatures could damage the protein content and also irreversibly degrade the sample.

Rehydration has to be considered as an indicator of the structural restoration after drying, as it influences both mechanical and morphological properties. In our study we did not observed substantially differences between the rehydration time and the weight recovery between the two processes. Moreover, the drying and rehydration steps did not influence the ECM organization; H\&E staining revealed the preservation of a multi-layered macro-architecture. In addition, MT and $\mathrm{AB}$ staining showed collagen maintenance and no apparent $\mathrm{GAG}$ reductions after the $\mathrm{SC}-\mathrm{CO}_{2}$ and the EtOH+SC- $\mathrm{CO}_{2}$ treatments. This is an important result since Totonelli et al. previously revealed a significant GAG decreasing associated to the loss of the glandular structure after the DET treatment [18].

The SC- $\mathrm{CO}_{2}$ and $\mathrm{EtOH}+\mathrm{SC}-\mathrm{CO}_{2}$ treatment generated diffused micro-porosity with no visible histological damage to the ECM structure layers. The load/width-strain curves did not show significant changes before and after the treatment in case of both longitudinal and transversal sections. The porosity was higher for the EtOH+ $\mathrm{SC}-\mathrm{CO}_{2}$ treatment as expected; indeed the cosolvent influence positively the $\mathrm{SC}-\mathrm{CO}_{2}$ solubility. On venting the $\mathrm{SC}-\mathrm{CO}_{2}$ by depressurization, a supersaturation of $\mathrm{CO}_{2}$ is generated and hence nucleation and growth occurred into the matrix. No significant difference between depressurization time was found, probably because of the low solubilisation of $\mathrm{SC}-\mathrm{CO}_{2}$ in the polymeric matrix.

To ensure the maintenance of the cell adhesion protein and the absence of chemical residuals from the previous DET, we performed a set of proof of concept cell culture repopulation experiments using the rehydrated matrix. hBM-MSCs were viable and proliferating at the surface after 7 days of culture for both the treatments. Since $\mathrm{SC}-\mathrm{CO}_{2}$ is largely used as extracting component, we had 
evidence that $\mathrm{SC}-\mathrm{CO}_{2}$ could be used also to eliminate toxic chemical residual from the decellularization process. We supposed that an extensive removal of chemicals from DET could not be guaranteed only by the use of traditional washing method due to the thickness and low porosity of the oesophagus samples. We showed that cell cultured through the conditioned media from the untreated samples were less viable compared to those from treated samples. These preliminary experiments on viability are very important for the development of artificial organs for transplantation. Human BM-MSCs are a good model for a preliminary study of cell viability because of their capability to differenziate in several different cell type, in particular myocytes and fibroblast. Moreover BM-MSCs are one of the most promising cell source for cell therapy and are largely used in many trials.

SC- $\mathrm{CO}_{2}$ showed attractive properties in the development of a dry scaffold with no substantial alteration of structural, mechanical and biological properties after rehydration. Both treatments here presented allowed to obtain dry matrices but the $\mathrm{EtOH}+\mathrm{SC}-\mathrm{CO}_{2}$ method allows a faster drying with a higher porosity rate. Additional studies are needed in order to investigate the possibility to extract other volatile component other than water with both the treatment. The generation of controlled pores in size and number could be a useful property for further cell scaffold re-seeding prior implantation. However, further studies are needed in order to confirm the advantages of porosity in the ECM for cell scaffold repopulation. Specific studies are also required in order to increase the cell repopulation inside the acellular matrix. The use of a specific co-culture in vitro model or chemo-attractive component could give substantial advantages for cell repopulation. Moreover, the effect of $\mathrm{SC}-\mathrm{CO}_{2}$ sterilization on the microbial content of the natural derived tissue is also important to be explored in the perspective of a standard clinical grade scaffold. Supplementary studies are needed and they require aged dried samples in order to control the maintenance of biological and mechanical properties. 


\section{CONCLUSION}

Taking advantage of SC- $\mathrm{CO}_{2}$ drying process, we obtained a dry decellularized scaffold that could be used for the development of organ/tissue substitution. A dry acellular matrix was obtained coupling SC- $\mathrm{CO}_{2}$ drying technique to a decellularized oesophagus obtained via DET. The treatment allowed the obtainment of a dry scaffold with preserved both its macro and micro structure, which also showed good biocompatible properties in terms of cell culture viability in vitro. The results of the present study clearly demonstrated that $\mathrm{SC}-\mathrm{CO}_{2}$ treatment represents a promising innovative technique for the preparation of novel organ/tissue substitution a-toxic, in absolute dry conditions and with a suitable structure for cell repopulation. This study, which focused for the first time on oesophagus decellularized scaffold, could provide a novel approach to derive a standard technique for the preservation of other natural-derived scaffolds.

\section{ACKNOWLEDGEMENTS}

The research leading to these results received funding from Cassa di Risparmio di Trento e Rovereto (CaRiTRo) within the research project "Supercritical decellularization of engineered tissues for clinical application", biomedical science section, 2013. PDC is supported by NIHR Professorship and the Catapult Cell Therapy, UK. MP is supported by Foundation Institute of Pediatric Research Città della Speranza, Grant 12/01. NMP is supported by the European Research Council (ERC StG Ideas 2011 BIHSNAM no. 279985 on 'Bio-Inspired hierarchical supernanomaterials', ERC PoC 2013 KNOTOUGH no. 632277 on 'Super-tough knotted fibres', ERC PoC 2015 SILKENE no. 693670 on 'Bionic silk with graphene or other nanomaterials spun by silkworms') and by the European Commission under the Graphene Flagship (WP14 'Polymer Composites', no. 696656) and by the Provincia Autonoma di Trento ("Graphene Nanocomposites", n. S116/2012-242637 and reg.delib. n. 2266). NE thanks Lorenza Lazzari for the donation of BMMSCs from the Cell Factory Bank (Milan-Italy). 


\section{References}

[1] Spitz L, Kiely E, Pierro A. Gastric transposition in children-a 21-yearexperience. J Pediatr Surg. 2004; 39:276-281.

[2] Ferlay, J., et al. "GLOBOCAN 2012 v1. 0, Cancer Incidence and Mortality Worldwide: IARC CancerBase No. 11. Lyon, France: International Agency for Research on Cancer; 2013." Last accessed April (2014).

[3] Spitz L., Esophageal replacement: Overcoming the need Journal of Pediatric Surgery 2014; 49: 849-852.

[4] Hamza A.F. Colonic replacement in cases of esophageal atresia. Semin Pediatr Surg. 2009; $18: 40-43$.

[5] Cowles Robert A., Arnold G. Coran. Gastric transposition in infants and children Pediatr. Surg. Int. 2010; 26: 1129-1134.

[6] Stephen F. Badylak, S. F., D.V.M., Ph.D., M.D., David A. Vorp, Ph.D., Alan R. Spievack, M.D., Abby Simmons-Byrd, R.V.T., Joseph Hanke, Donald O. Freytes, M.S., Anil Thapa, M.S., Thomas W. Gilbert, and Alejandro Nieponice, M.D. Esophageal Reconstruction with ECM and Muscle Tissue in a Dog Model. Journal of Surgical Research 128, 87-97 (2005).

[7] Ron, Ori, Paolo De Coppi, and Agostino Pierro. "The surgical approach to esophageal atresia repair and the management of long-gap atresia: results of a survey." Seminars in pediatric surgery. Vol. 18. No. 1. WB Saunders, 2009.

[8] Maghsoudlou P., BSc (Hons.),Eaton S., De Coppi P. Tissue engineering of the esophagus. Seminars in Pediatric Surgery 2014; 23:127-134.

[9] Watanabe M, Sekine K, Hori Y., Artificial esophagus with peristaltic movement. ASAIO J. 2005;5 1(2):158-161. 

middle third of the esophagus with a silicone rubber prosthesis .An experiment and clinical study. Dis Chest. 1963; 43(5):464-475.

[11] Takimoto Y, Nakamura T, Teramachi M, Kiyotani T, Shimizu Y. Replacement of long segments of the esophagus with a collagen-silicone composite tube. ASAIO J 1995; 41:M605-M608.

[12] Yamamoto Y., Nakamura T., Shimizu Y., Matsumoto K., Takimoto Y.,Liu Y., Ueda H., Seikine T. and Tamura N. Intrathoracic esophageal replacement with a collagen sponge - silicone double layer tube: evaluation of omental-pedicle wrapping and prolonged placement of an inner stent. ASAIO Journal 2000; 46:734-739.

[13] Uygun, Basak E., et al. "Organ reengineering through development of a transplantable recellularized liver graft using decellularized liver matrix." Nature medicine 16.7 (2010): 814-820.

[14] Sullivan, David C., et al. "Decellularization methods of porcine kidneys for whole organ engineering using a high-throughput system." Biomaterials 33.31 (2012): 7756-7764.

[15] Gilbert T.W., SellaroT.L.,Badylak S.F. Decellularization of tissues and organs. Biomaterials. 2006; 27(19):3675-3683.

[16] Petersen, Thomas H., et al. "Tissue-engineered lungs for in vivo implantation." Science 329.5991 (2010): 538-541.

[17] Ott, Harald C., et al. "Perfusion-decellularized matrix: using nature's platform to engineer a bioartificial heart." Nature medicine 14.2 (2008): 213-221.

[18] Totonelli G, Maghsoudlou P, Georgiades F, Garriboli M, Koshy K, Turmaine M, Ashworth M, Sebire NJ, Pierro A, Eaton S, De Coppi P. Detergent enzymatic treatment for the development of a natural acellular matrix for oesophageal regeneration. Pediatric Surgery International, 2013, 29, 87-95. 

engineering: toxicological effects of residual detergents on human endothelial cells." Artificial organs 34.3 (2010): 206-210.

[20] Baiguera, Silvia, et al. "Long-term changes to in vitro preserved bioengineered human trachea and their implications for decellularized tissues." Biomaterials 33.14 (2012): $3662-3672$.

[21] Bonenfant, Nicholas R., et al. "The effects of storage and sterilization on decellularized and re-cellularized whole lung." Biomaterials 34.13 (2013): 3231-3245.

[22] Van Arsdel, Wallace B., Michael J. Copley, and A. I. Morgan Jr. Food dehydration. Vol. 1. Drying methods and phenomena. No. Ed. 2. 1973.

[23] Brown Z.K. et al. Drying of foods using supercritical carbon dioxide Investigations with carrot. Innovative Food Science and Emerging Technologies 2008; 9: 280-289.

[24] Floren M., Spilimbergo S., Motta A., Migliaresi C. Porous poly(D,L-lactic acid ) foams with tunable structure and mechanical anisotropy prepared by supercritical carbon dioxide. Journal of Biomedical Materials Research Part B: Applied Biomaterials, 2011: 338349.

[25] Migliaresi, C., M. L. Floren, S. Spilimbergo, and A. Motta. "Novel preparation of silk hydrogels under high pressure carbon dioxide." In JOURNAL OF TISSUE ENGINEERING AND REGENERATIVE MEDICINE, vol. 6, pp. 178-178. 111 RIVER ST, HOBOKEN 07030-5774, NJ USA: WILEY-BLACKWELL, 2012.

[26] Tsivintzelis, I., Marras, S. I., Zuburtikudis, I., \& Panayiotou, C. (2007). Porous poly (l-lactic acid) nanocomposite scaffolds prepared by phase inversion using supercritical CO 2 as antisolvent. Polymer, 48(21), 6311-6318. 

scaffolds for tissue engineering by supercritical immersion precipitation. The Journal of Supercritical Fluids, 49(2), 279-285.

[28] Reverchon, E., \& Cardea, S. (2012). Supercritical fluids in 3-D tissue engineering. The Journal of Supercritical Fluids, 69, 97-107.

[29] Spilimbergo S, Bertucco A. Non-thermal bacteria inactivation with dense CO2. Biotechnol Bioeng 2003; 84: 627-638.

[30] Ferrentino G., and Spilimbergo, S. High pressure carbon dioxide pasteurization of solid foods: current knowledge and future outlooks, Trends in Food Science \& Technology, 2011, $22(8): 427-441$

Qui Q.Q., et al., Inactivation of Bacterial Spores and Viruses in Biological Material Using Supercritical Carbon Dioxide with sterilant, Journal of Biomedical Materials research PART B-Applied Biomaterials, 2009, 91B: 572-578. A. (1993). Cosolvent interactions in supercritical fluid solutions. AIChE Journal, 39(2), $235-248$. of some organic acids in supercritical $\mathrm{CO}_{2}$ with polar solvents. Journal of Supercritical Fluids, 11(1-2), 53-60.

[34] Dodds WS, Stutzman LF, Sollami BJ. 1956. Carbon dioxide solubility in water. Ind Eng Chem 1:92-5.

[35] Spilimbergo S, Bertucco A, Basso G, Bertoloni G. 2005. Determination of extracellular and intracellular $\mathrm{pH}$ of Bacillus subtilis suspension under $\mathrm{CO}_{2}$ treatment. Biotechnol Bioeng 92:447-51. 
[36] Garcìa-Gonzalez, L., Geeraerd, A.H., Spilimbergo, S., Elst, K., Van Ginneken, L., Debevere, J., Van Impe, J.F., \& Devlieghere, F. (2007). High pressure carbon dioxide inactivation of microorganisms in foods: The past, the present and the future. International Journal of Food Microbiology 117, 1-28.

[37] Macchiarini, Paolo, et al. "Clinical transplantation of a tissue-engineered airway." The Lancet 372.9655 (2008): 2023-2030.

[38] Elliott, Martin J., et al. "Stem-cell-based, tissue engineered tracheal replacement in a child: a 2-year follow-up study." The Lancet 380.9846 (2012): 994-1000.

[39] Ott, Harald C., et al. "Perfusion-decellularized matrix: using nature's platform to engineer a bioartificial heart." Nature medicine 14.2 (2008): 213-221.

[40] Totonelli, Giorgia, et al. "A rat decellularized small bowel scaffold that preserves villus-crypt architecture for intestinal regeneration." Biomaterials 33.12 (2012): 3401-3410. 
Table 1. Summary of the experimental conditions applied at at $35^{\circ} \mathrm{C}$ and $10 \mathrm{MPa}$.

\begin{tabular}{|l|l|}
\hline Treatment & Conditions \\
\hline $\mathrm{T}_{1}$ & $60 \min \mathrm{SC}-\mathrm{CO}_{2}$ \\
\hline $\mathrm{T}_{2}$ & $120 \min \mathrm{SC}-\mathrm{CO}_{2}$ \\
\hline $\mathrm{T}_{3}$ & $80 \min \mathrm{EtOH}+15 \min \mathrm{SC}-\mathrm{CO}_{2}$ \\
\hline $\mathrm{T}_{4}$ & $80 \min \mathrm{EtOH}+30 \min \mathrm{SC}-\mathrm{CO}_{2}$ \\
\hline
\end{tabular}



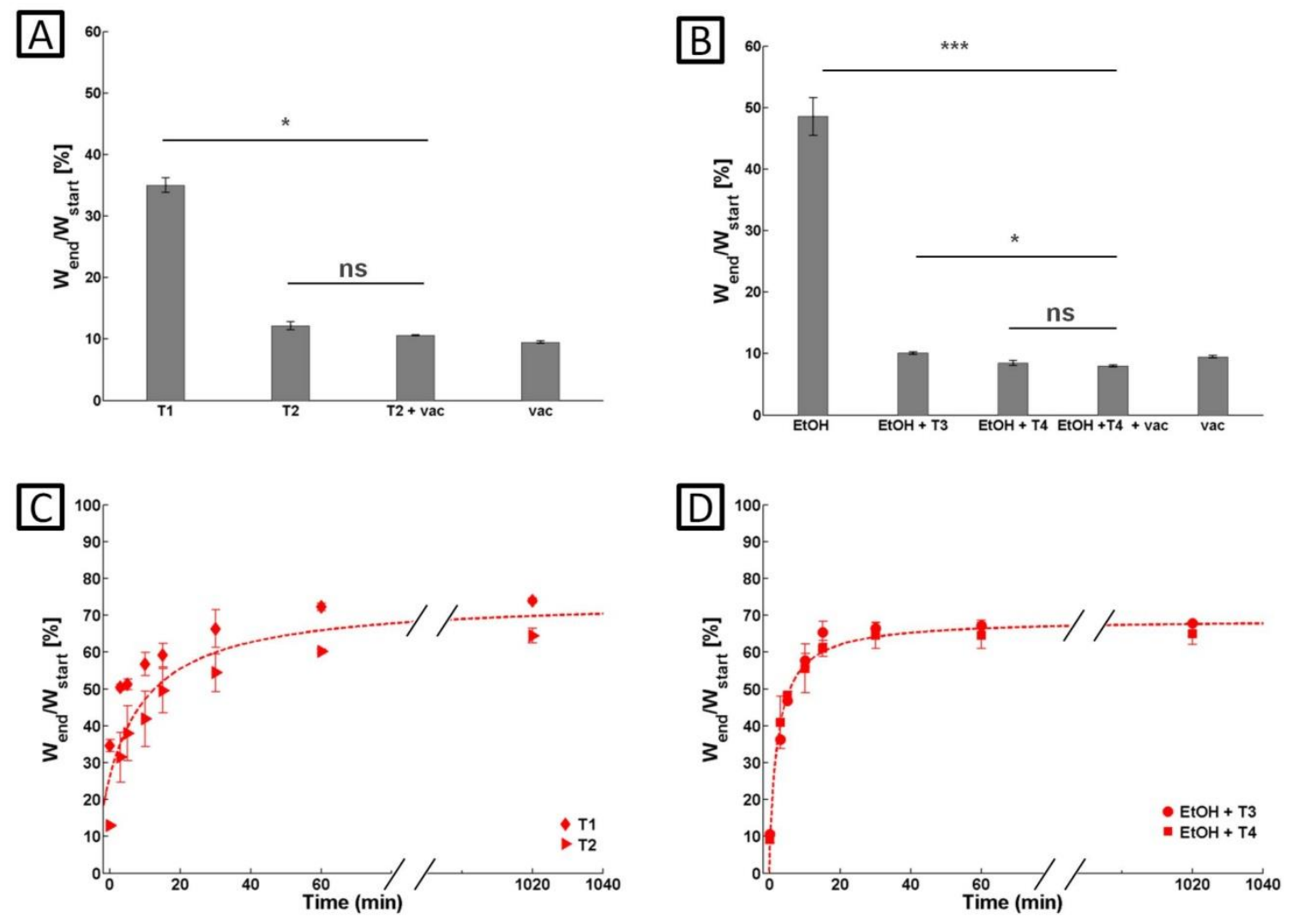

Figure 1. (A) Loss of weight percentage of ECM after $\mathrm{SC}-\mathrm{CO}_{2}$ treatments $\left(\mathrm{T}_{1}\right.$ for $60 \mathrm{~min}$ and $\mathrm{T}_{2}$ for $120 \mathrm{~min}$ ), $\mathrm{SC}-\mathrm{CO}_{2}$ treatment for $120 \mathrm{~min}+$ vacuum drying for $12 \mathrm{~h}\left(\mathrm{~T}_{2}+\right.$ vacuum$)$, and vacuum drying for $12 \mathrm{~h}$ as control (vacuum); (B) Loss of weight percentage of ECM after EtOH dehydration (80 min), EtOH dehydration $\left(80 \mathrm{~min}\right.$ ) and $\mathrm{SC}-\mathrm{CO}_{2}$ treatments $\left(\mathrm{T}_{3}\right.$ for $15 \mathrm{~min}$ and $\mathrm{T}_{4}$ for $30 \mathrm{~min}$ ), EtOH dehydration $(80 \mathrm{~min})+\mathrm{SC}-\mathrm{CO}_{2}$ for $30 \mathrm{~min}+$ vacuum drying for $12 \mathrm{~h}\left(\mathrm{~T}_{4}+\right.$ vacuum$)$, and vacuum drying for $12 \mathrm{~h}$ as control (vacuum); (C) Rehydration kinetics of dried samples after SC$\mathrm{CO}_{2}$ treatment $(\diamond, 60 \mathrm{~min}(\mathrm{~T} 1) ;-, 120 \mathrm{~min}(\mathrm{~T} 2))$; (D) and $\mathrm{EtOH}(80 \mathrm{~min})+\mathrm{SC}-\mathrm{CO}_{2}$ treatment $(\bullet$, $15 \min (\mathrm{T} 3) ; \mathbf{m}, 30 \min (\mathrm{T} 4))$. 


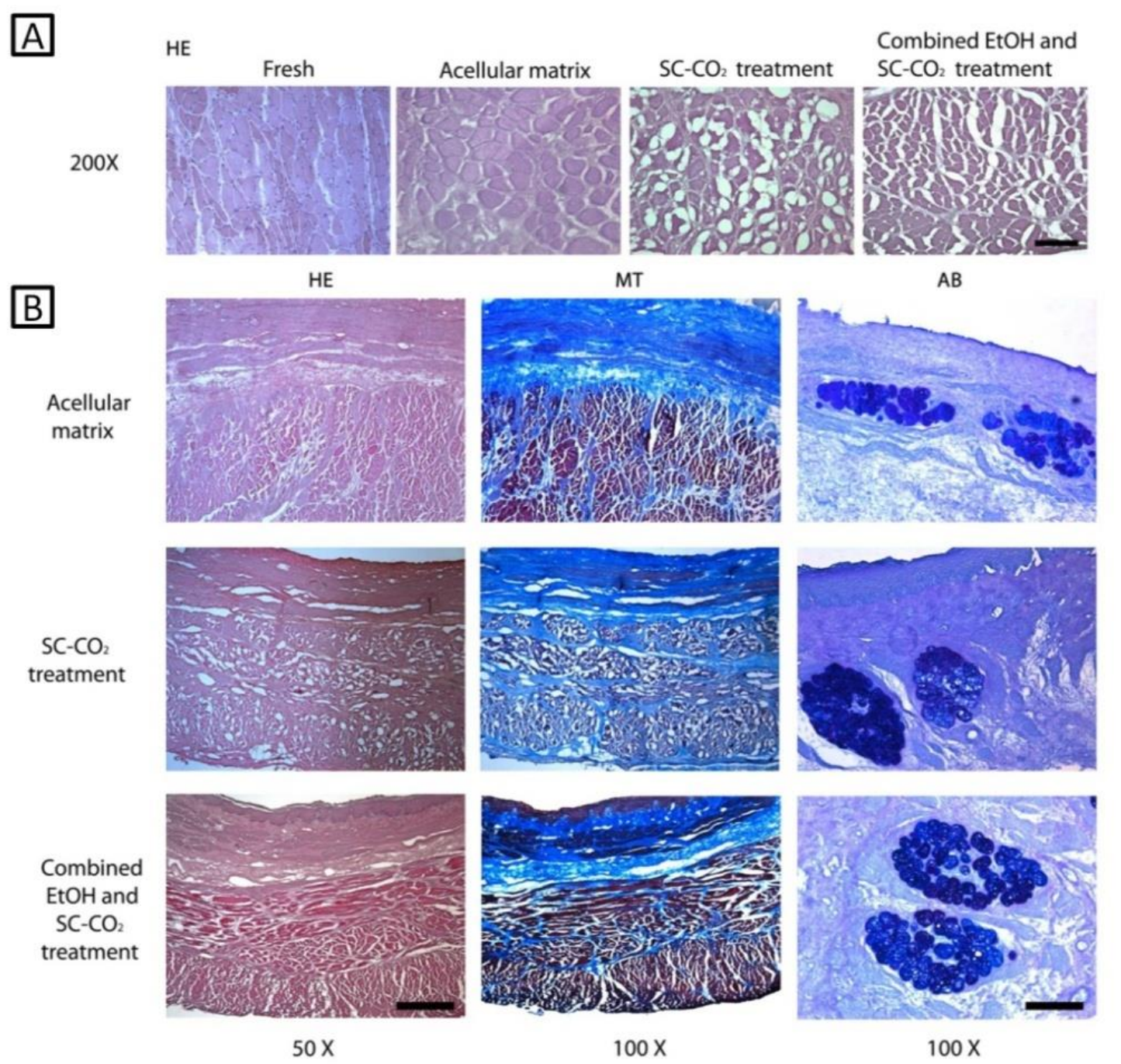

Figure 2. Histological analysis. A). H\&E staining confirmed the absence of intact nuclei and the generation of a micro-porosity after $\mathrm{SC}-\mathrm{CO}_{2}$ treatment $(120 \mathrm{~min})$ and $\mathrm{EtOH}(80 \mathrm{~min})+\mathrm{SC}-\mathrm{CO}_{2}$ treatment (15 $\mathrm{min})(\mathrm{B}) . \mathrm{H} \& \mathrm{E}$ staining demonstrated the maintenance of oesophageal architecture with the presence of mucosa, submucosa and muscular layers. MT staining confirmed the preservation of collagen in the intermuscular septa of muscularis externa, submucosa and lamina propria. AB staining did not show GAG loss (c). Scale bars $100 \mu \mathrm{m}$ for $200 \mathrm{X}, 250 \mu \mathrm{m}$ for $100 \mathrm{X}$, $500 \mu \mathrm{m}$ for $50 \mathrm{X}$. 

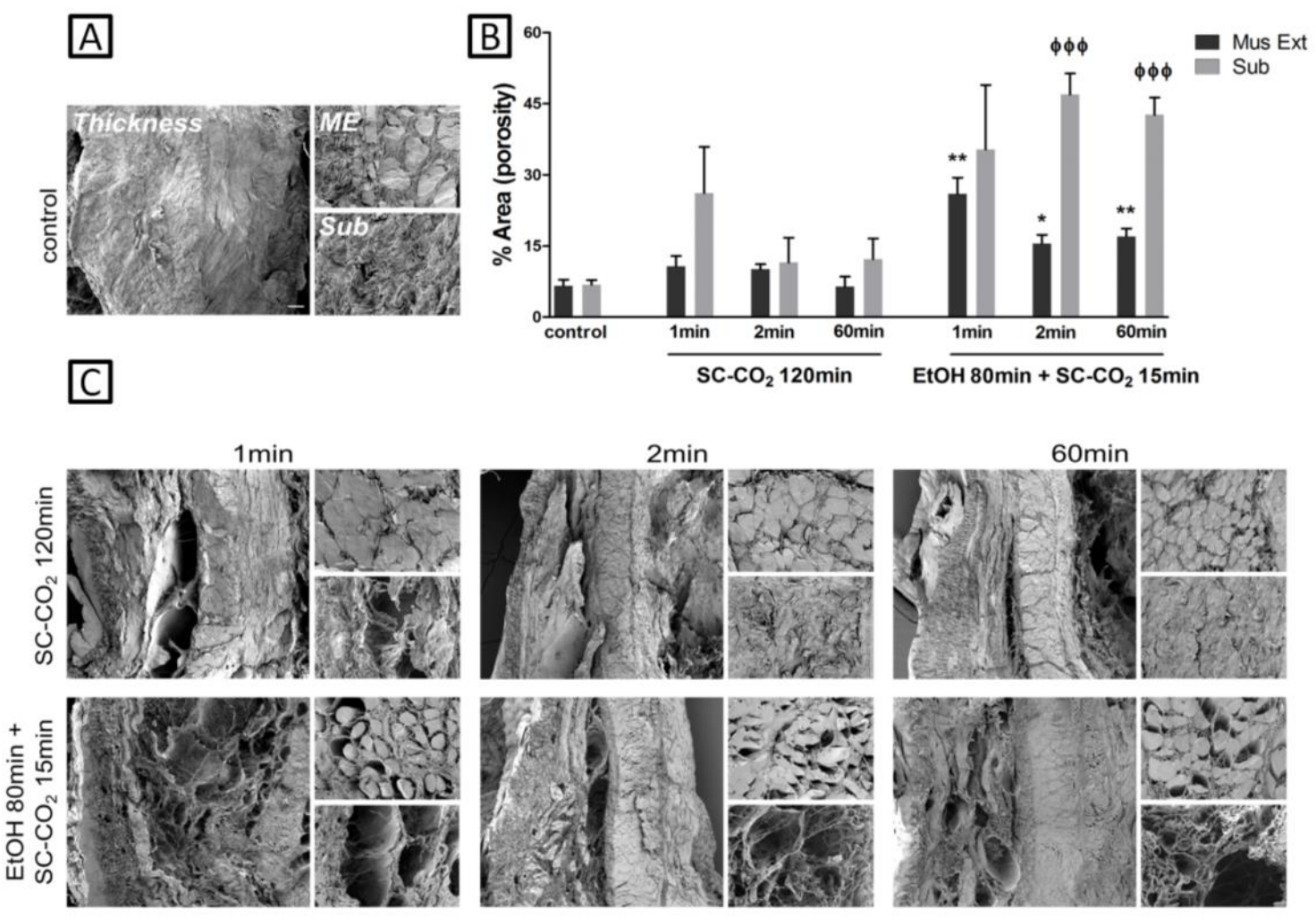

Figure 3. Porosity analysis. (A) SEM images representing the thickness of a decellularized and not dried sample (control) divided in Muscularis External (ME) and Submucosa Sub. (B) Percentages of porosity for different treatments and depressurization time (1 min, $2 \mathrm{~min} 60 \mathrm{~min}$ ) for Muscularis Externa and Submucosa (average of 3 samples and >5 measurements), (C) SEM images for different treatment and depressurization times. 

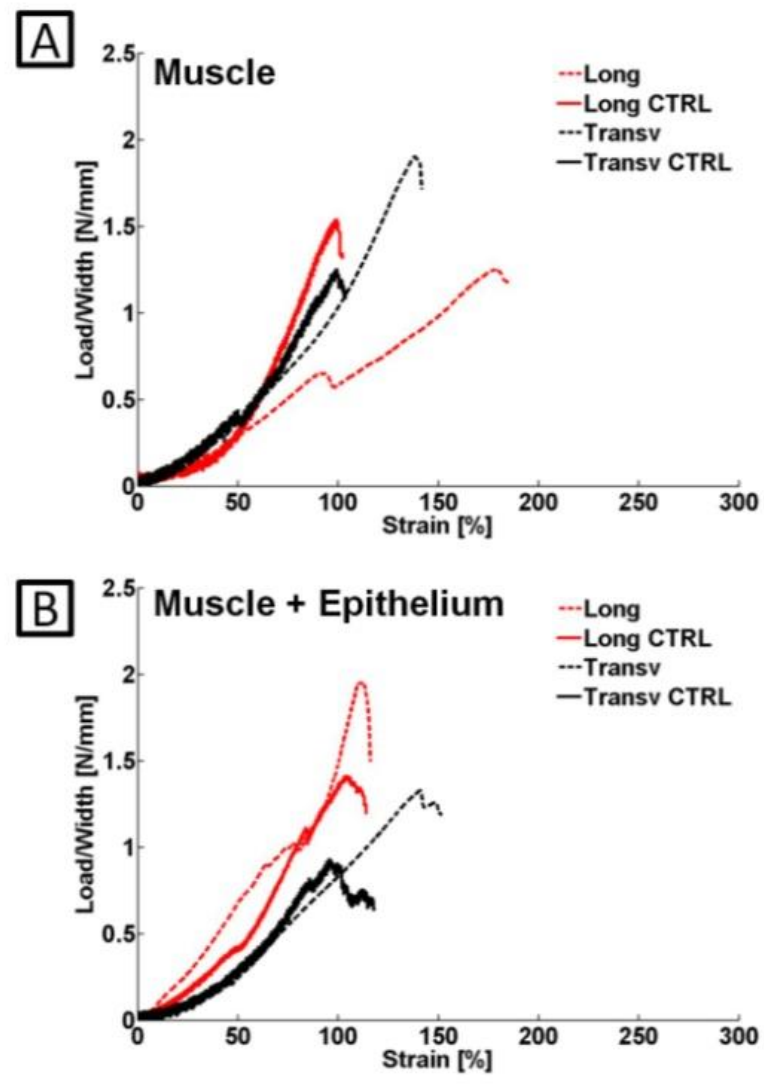

Figure 4: Load/width-strain curves derived for transversal (black) and longitudinal (red) samples consisting of muscle (A) and of both epithelium and muscle (B) extracted from either treated (dotted line) or control tissue (solid line) referred to a not dried decellularized tissue. 

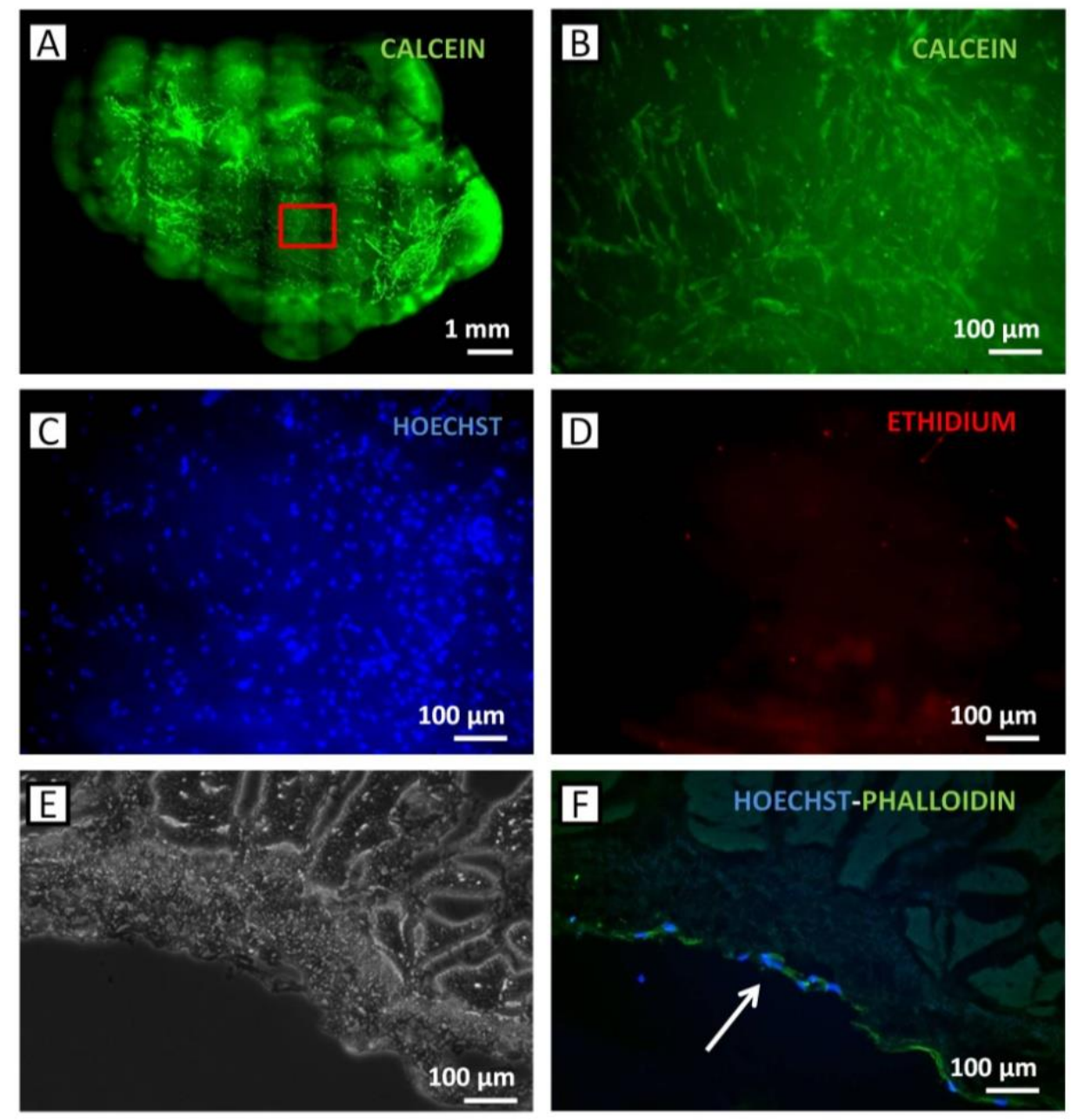

Figure 5: Cell Repopulation. Live and dead analysis of the sample surface after 7 days of culture with BM-MSc (A) merged pictures of calcein and red enlargement in green Calcein (B), Nuclei in blue with Hoechst (C) and Ethidium in red (D). Images of Slices in Phase (E) and with merged nuclei (blue) and phalloidin (green). Sample after treatment with EtOH and $\mathrm{SC}-\mathrm{CO}_{2}$. 


\section{Supplementary Information}

Title

Dry Acellular Oesophageal Matrix Prepared By Supercritical Carbon Dioxide

\section{Authors}

Alessandro Zambon ${ }^{1,2,3 \dagger}$, Massimo Vetralla ${ }^{1,2,3 \dagger}$, Luca Urbani ${ }^{4}$, Maria F. Pantano ${ }^{5}$, Giovanna Ferrentino $^{1}$, Michela Pozzobon ${ }^{6}$, Nicola M. Pugno ${ }^{5,7,8}$, Paolo De Coppi ${ }^{4,6}$, Nicola Elvassore ${ }^{2,3}$, Sara Spilimbergo ${ }^{1,2^{*}}$

${ }^{1}$ Department of Industrial Engineering, University of Trento, via Sommarive, 9 - 38123 Povo, Trento, Italy

${ }^{2}$ Department of Industrial Engineering, University of Padova, via Marzolo 9-35131 Padova, Italy

${ }^{3}$ Venetian Institute of Molecular Medicine, Via Orus Giuseppe 2- 35129 Padova, Italy

${ }^{4}$ Stem Cells and Regenerative Medicine Section, Developmental Biology and Cancer Program, Institute of Child Health, University College London - 30 Guilford Street, WC1N 1EH London, United Kingdom

${ }^{5}$ Laboratory of Bio-inspired \& Graphene Nanomechanics, Department of Civil, Environmental and Mechanical Engineering, University of Trento, Via Mesiano 77, 38123 Trento, Italy

${ }^{6}$ Stem cells and regenerative medicine lab, Foundation Institute of Pediatric Research Città della Speranza- corso stati uniti 4, 35136 Padova, Italy

${ }^{7}$ Center for Materials and Microsystems, Fondazione Bruno Kessler, Via Sommarive 18, 38123 Povo (TN), Italy

${ }^{8}$ School of Engineering and Materials Science, Queen Mary University of London, Mile End Road, London E1 4NS, U.K.

* corresponding author sara.spilimbergo@ unipd.it FAX +390498275461

$\dagger$ these authors have contributed equally to this work 


\section{$\underline{\text { Table of contents }}$}

Figure S-1. $\mathrm{SC}-\mathrm{CO}_{2}$ set up .......................................... S-1

Figure S-2. Sample preparation ............................................. S-2

Figure S-3. Reduction sample weight by ethanol's resident time S-3

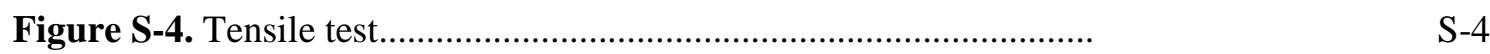

Figure S-5.microwell design and fabrication ........................................... S-5

Figure S-6 Sample insertion into microwell and culture........................................ S-6

Figure S-7 Toxicity ………………................ S-7

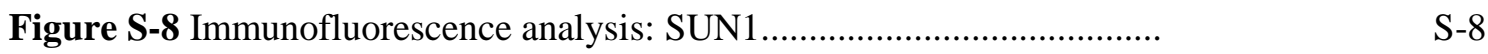

Figure S-9 $\mathrm{SC}-\mathrm{CO}_{2}$ culture at 7 days......................................... S-9

Figure S-10 Immunofluorescence analysis: Ki67 ........................................ S-10 


\section{Supplementary Fig. 1}
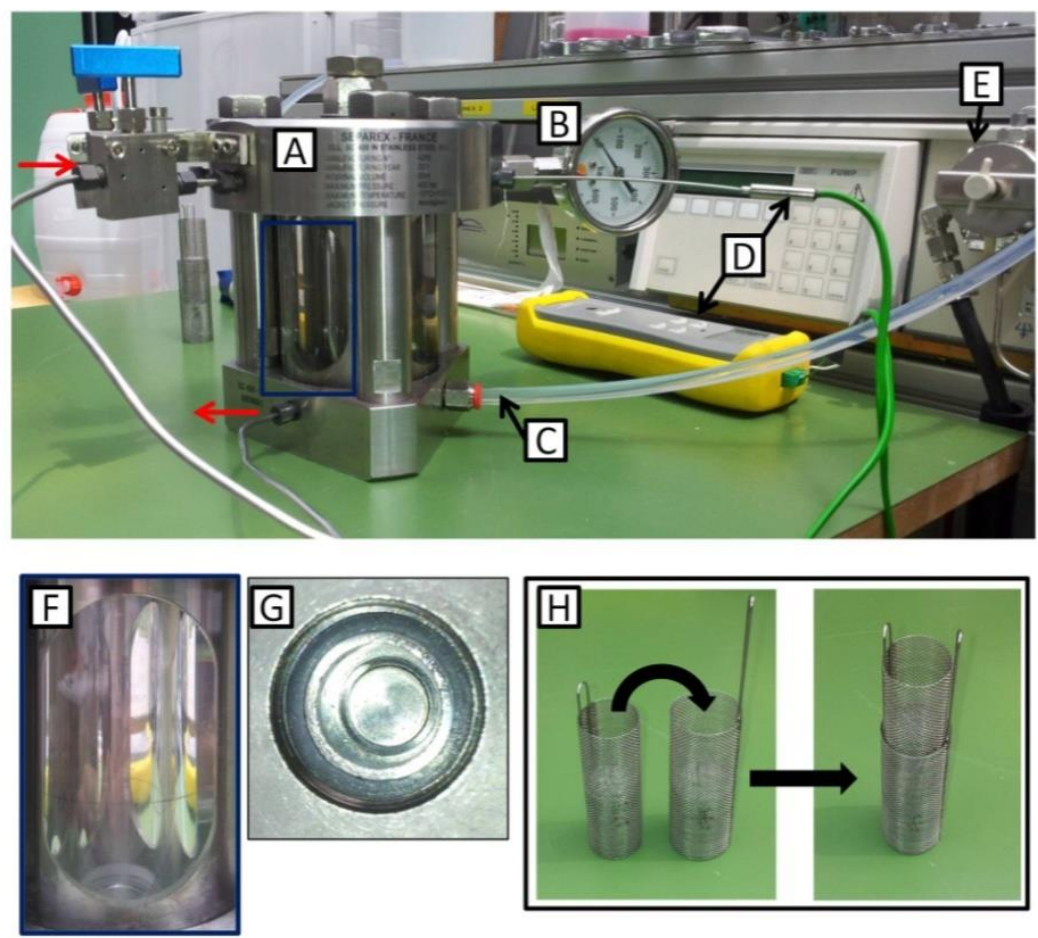

Experimental SC-CO2 set up. Image of the sapphire reactor (A) with two detailed views of the chamber (length (F) and diameter $(\mathrm{G})$ ), control pressure measurement (B), thermostatic liquid inlet in the external shell of the reactor (C), (D) Temperature measurement and controller, (E) $\mathrm{CO}_{2}$ pump; the red arrows show the $\mathrm{CO}_{2}$ inlet/outlet in the reactor. $(\mathrm{H})$ sequential images showing the small steel baskets used for sample placement inside the reactor. 


\section{Supplementary Fig. 2}

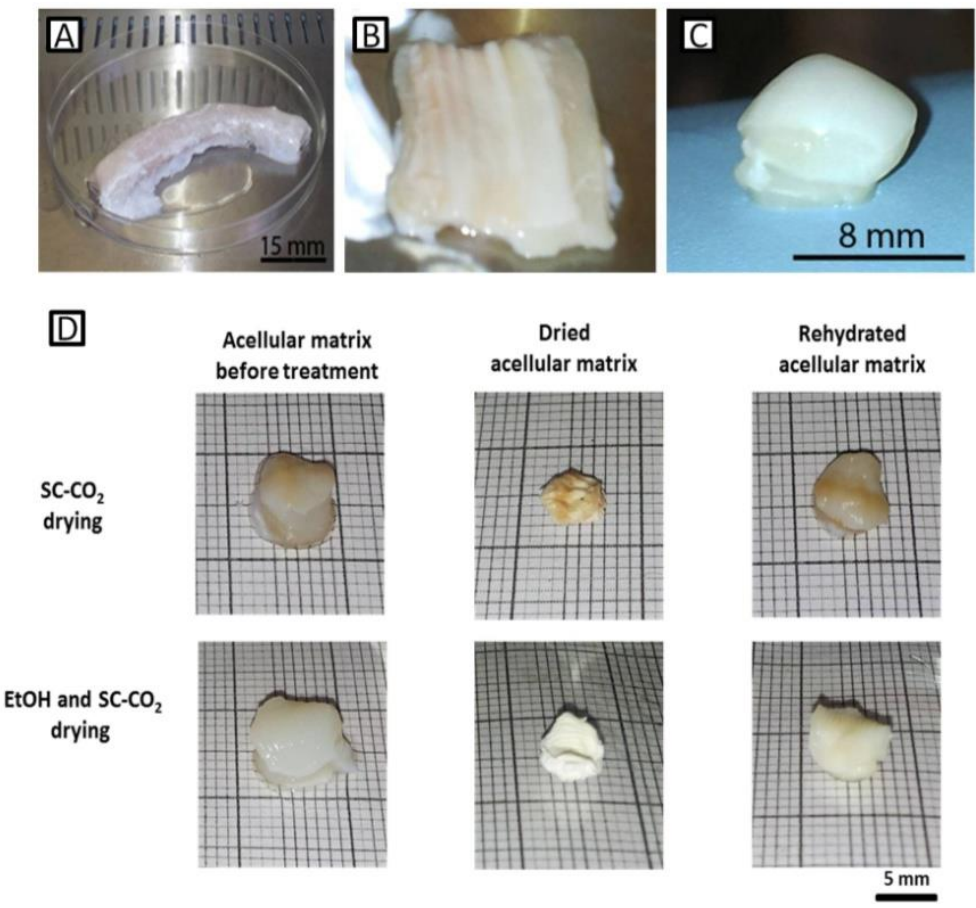

Sample preparation. Decellularized pig oesophagus before (A) and after (B) opening. (C) Planar sample $(\mathrm{D}=8 \mathrm{~mm})$ obtained after cutting. (D) Visual comparison between sample before and after SC- $\mathrm{CO}_{2}$ treatment and rehydration for the combined and single treatment. 
Supplementary Fig. 3

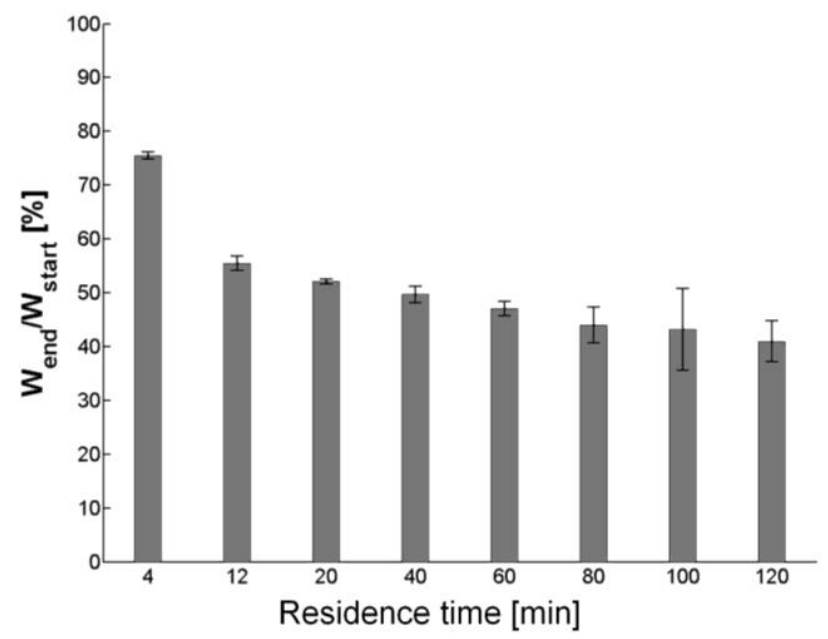

Dehydration with Ethanol. Reduction of sample's weight as a function of the residence time in EtOH solution. 
Supplementary Fig. 4

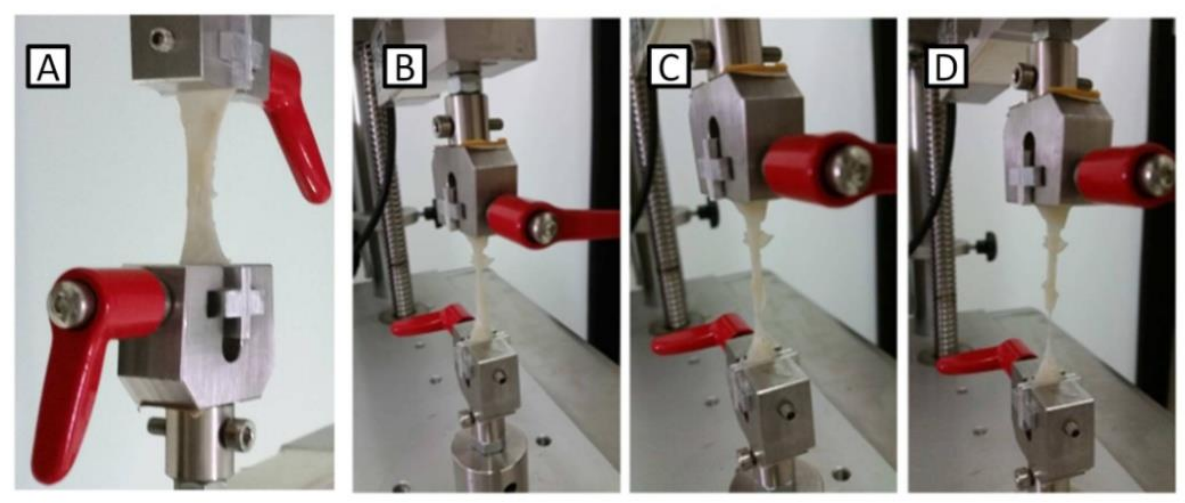

Tensile Test. (A) Picture of the sample fixed to the loading frame before applying the strain. (B),(C),(D) sequence of images during the test. 


\section{Supplementary Fig. 5}

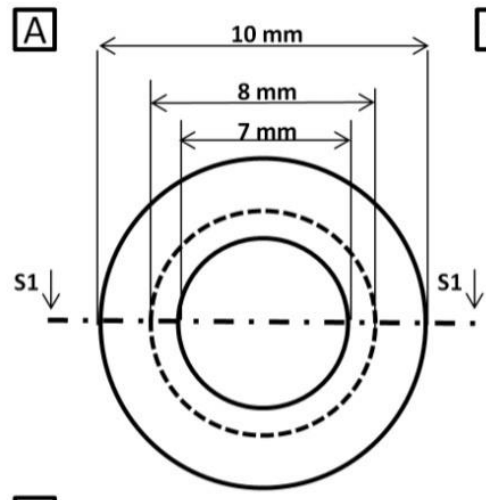

B

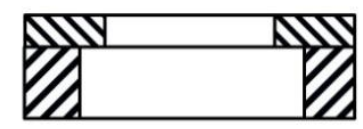

C.

PDMS
layer 1

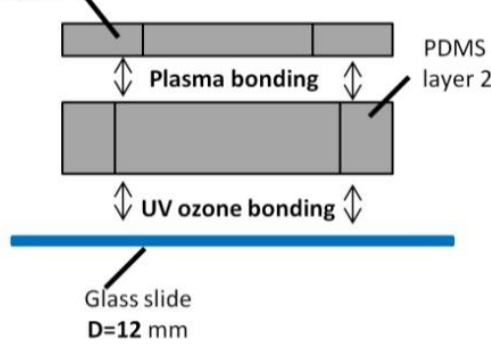

D

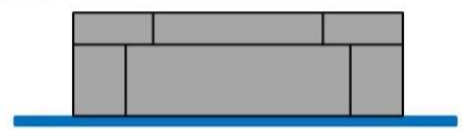

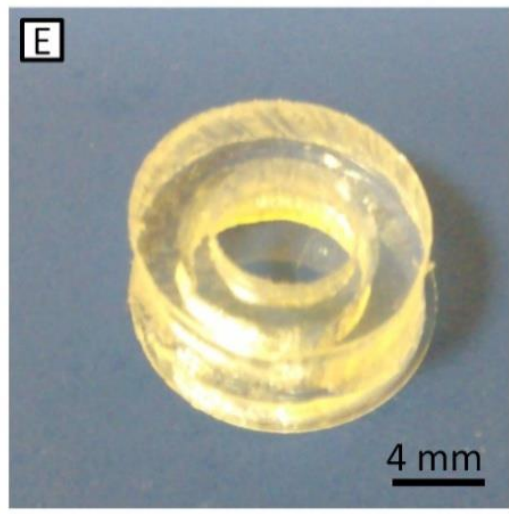

Microwell design and Fabrication. (A) Upper view and (B) lateral section S1 with quotes of the microwell. The PDMS was previously prepared with a 10:1 base to cure agent ratio and cured for at least 2 hours at $80^{\circ} \mathrm{C}$ inside a petri dish followed by cutting with scalpels. The microwell is composed of 2 PDMS layers bonded together via plasma treatment $(\mathrm{C})$. Then, the 2 layers were bounded to a circular $12 \mathrm{~mm}$ glass slice (menzel Glaser) via UV ozone (D). (E) reports a picture of the final microwell. Before using, the microwell was sterilized via autoclave. 
Supplementary Fig. 6

田
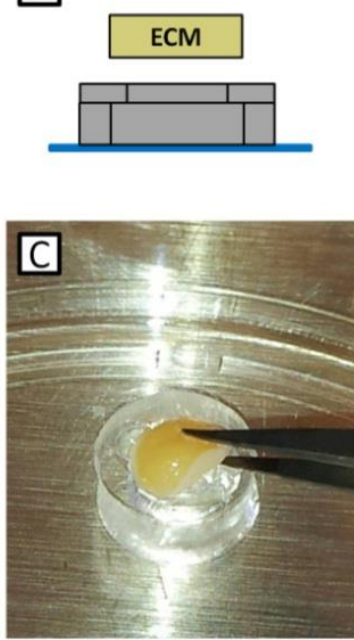

B
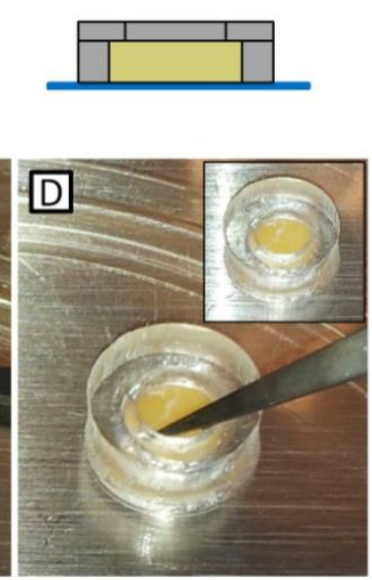

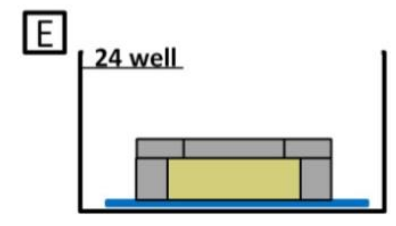

F Cells in medium $(150 \mu \mathrm{L})$

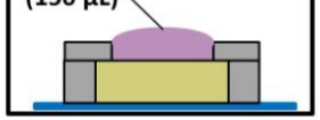

G

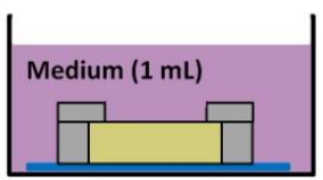

Sample insertion into microwell and cell culture. Schematic sequence of matrix insertion inside the microwell before cell seeding. (A) Sample was placed on the top of the microwell and using a sterile tweezers (C) put inside the well (B and D). Then the microwell was moved inside a 24 well (E) where cells were seeded (F) suspended in $150 \mu \mathrm{L}$ of medium. After $\mathrm{O} / \mathrm{N}$ incubation additional 1 $\mathrm{mL}$ of medium was added in each well, covering the entire surface of the microwell $(\mathrm{G})$. 


\section{Supplementary Fig. 7}
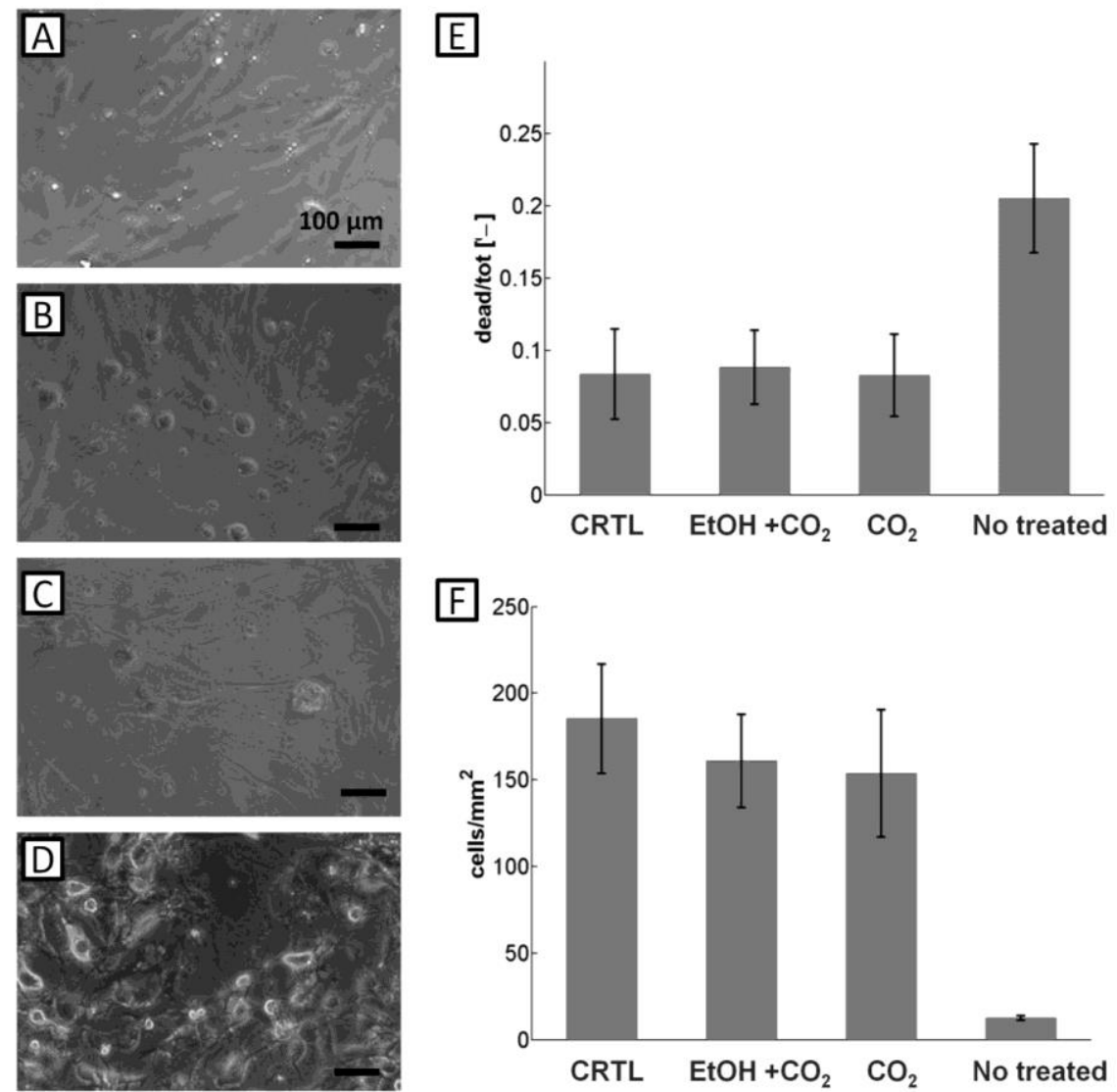

Toxicity. Phase images after 48 hours of hBM-MSCs with fresh media (CTRL) (A) and with conditioned media for sample treated with combined Ethanol and $\mathrm{SC}-\mathrm{CO}_{2}$ treatment (B), 120 minute $\mathrm{SC}-\mathrm{CO}_{2}(\mathrm{C})$ or no treated sample (D). (E) Live and dead analysis expressed as number of dead cells versus total cells at 48 hours for the same previous conditions. (F) Cells $/ \mathrm{mm}^{2}$ after 5 days of culture for the same conditions. 


\section{Supplementary Fig. 8}

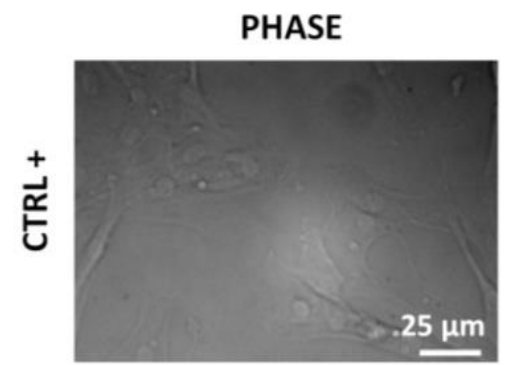

HOECHST SUN-1
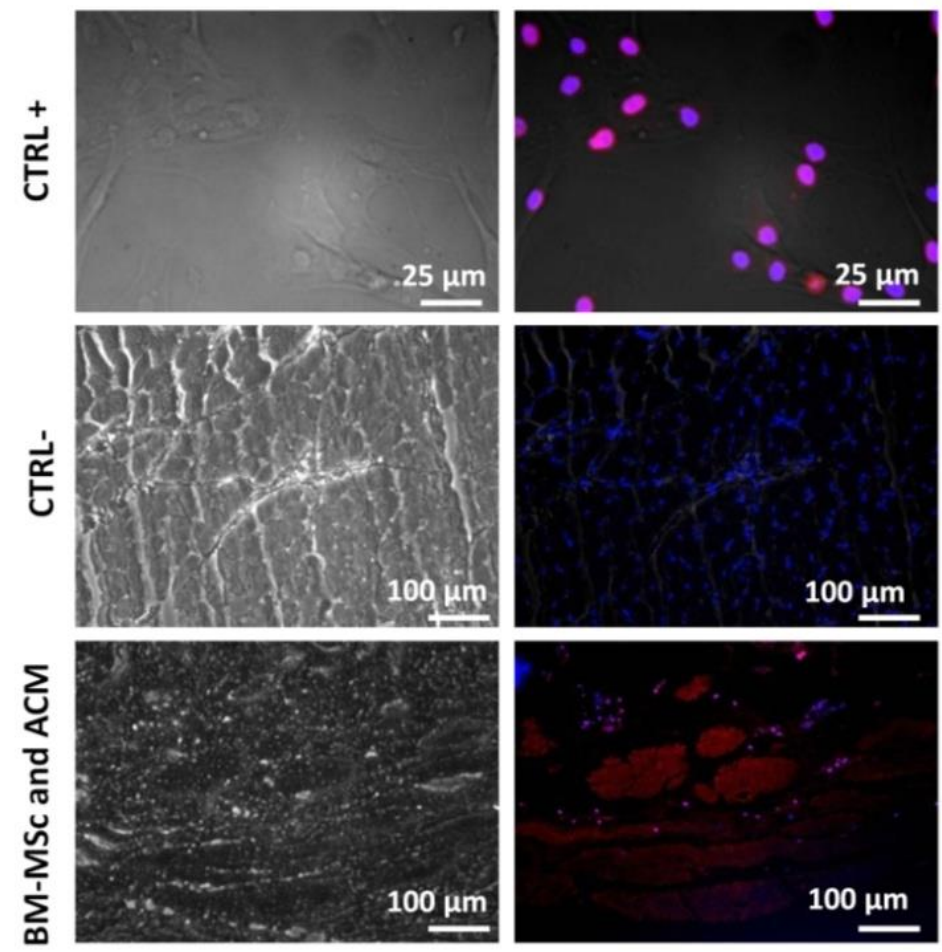

Immunofluorescence analysis: SUN1. Phase and fluorescence images for hBM-MSCs $(\mathrm{CTRL}+)$ on well, and slices of fresh porcine esophagus before decellularization (CTRL-) and decelularized and repopulated samples. Images at 40X for CTRL+ and 10X for the others. SUN1 is marked in red, while nuclei in blue. 


\section{Supplementary Fig. 9}

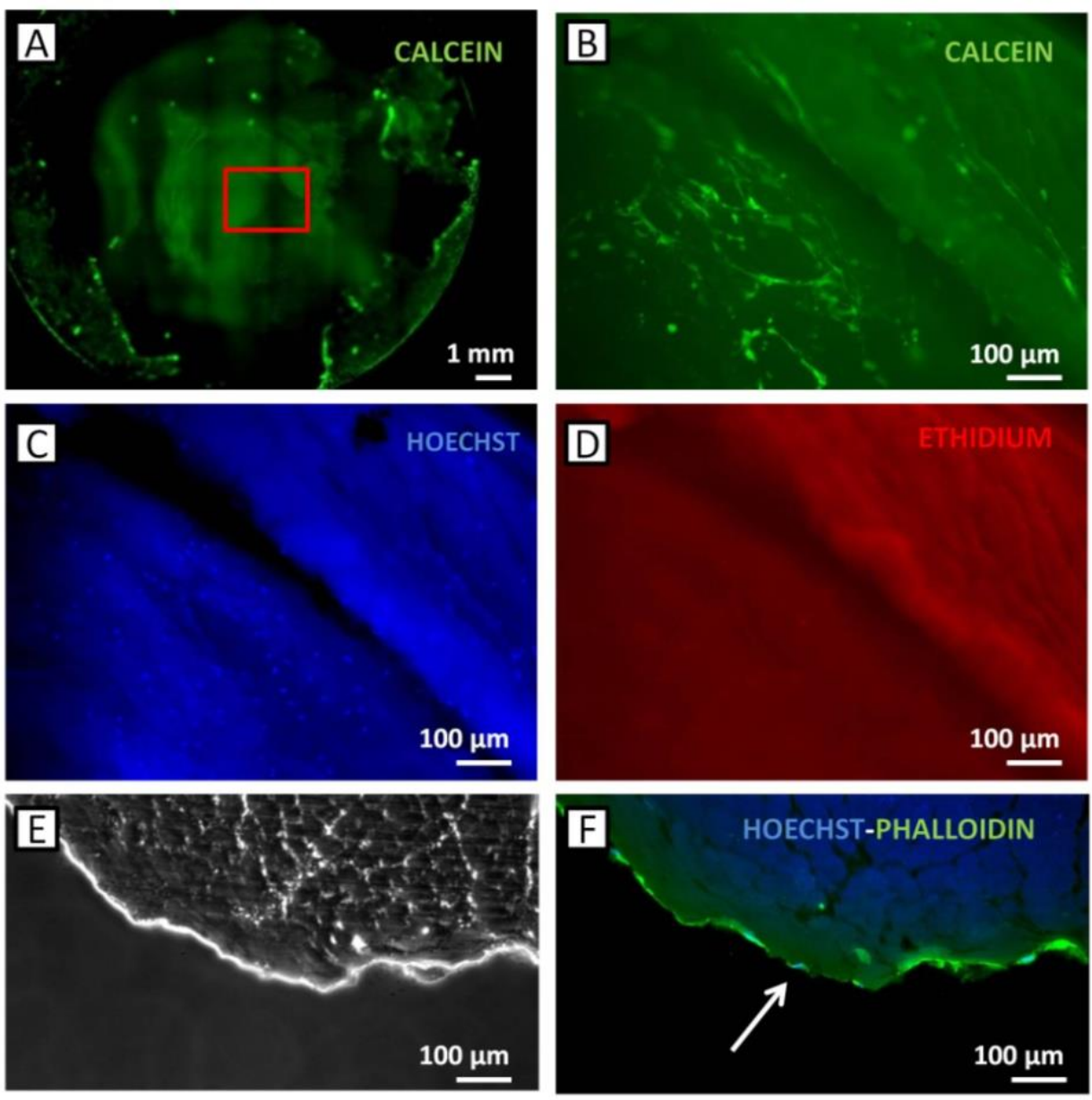

Cell repopulation at 7 days for sample treated with $\mathrm{SC}_{-} \mathrm{CO}_{2}$. Live and dead analysis of the sample surface after 7 days of culture with hBM-MSCs (A) merged pictures of calcein and red enlargement in green Calcein (B), Nuclei in blue with Hoechst (C) and Ethidium in red (D). Images of Slices in Phase (E) and with merged nuclei (blue) and phalloidin (green). 
Supplementary Fig. 10
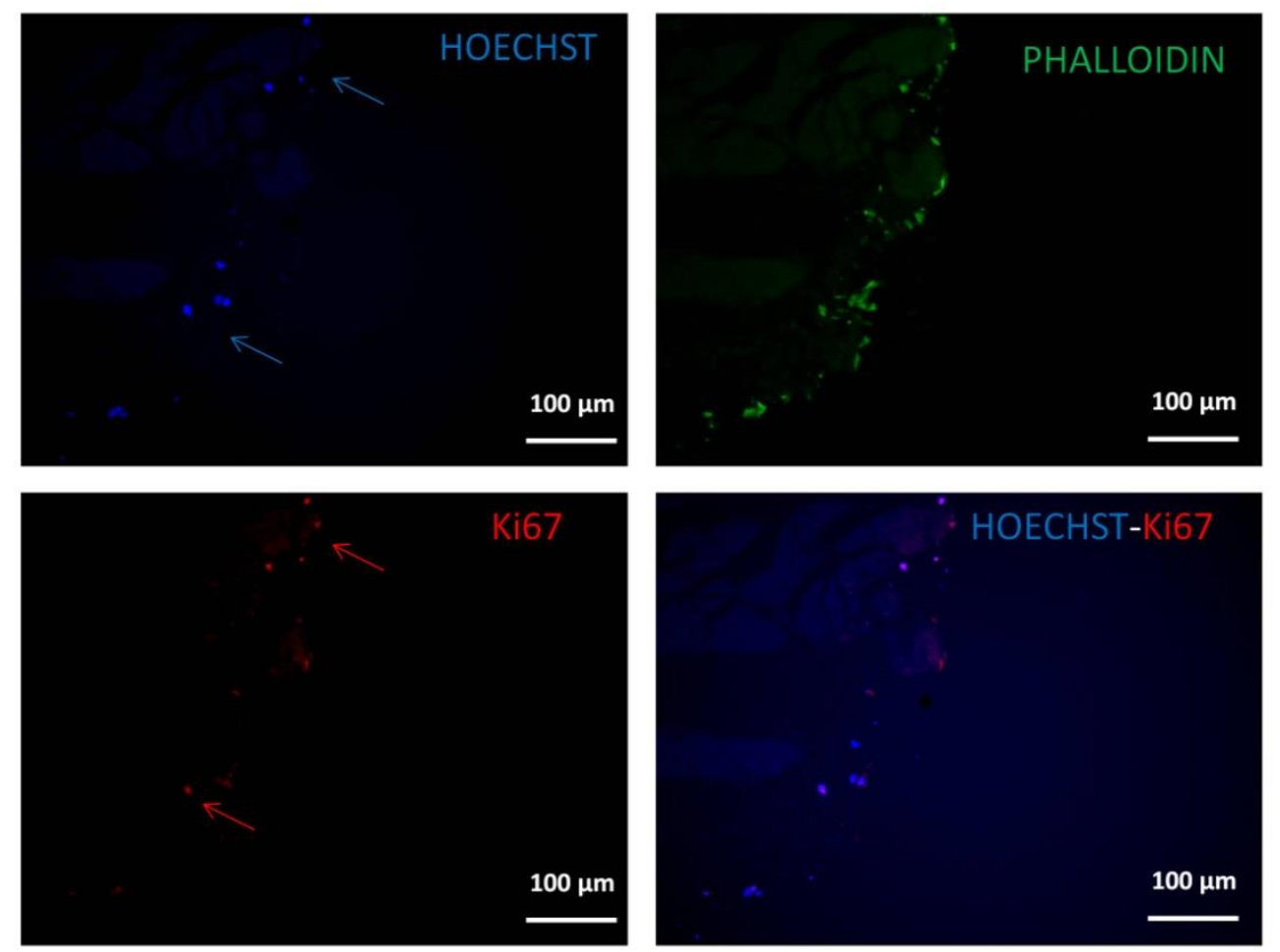

Immunofluorescence analysis: Ki67. Fluorescence images for hBM-MSCs after 7 days of culture with Hoechst (blue), Phalloidin (green) and Ki67 antibody (red). Merge image with Hoechst and Ki-67. 